\title{
Potential Role of Pleural Fluid Cytokine Profile in Myelomatous Pleural Effusions
}

\author{
Junhui $X u^{1,2, *}$ \\ Liang Gao ${ }^{1, *}$ \\ Miao Yan' \\ Bingjie Wang' \\ Zhengyang Song (D' \\ Huihui Liu' \\ Kunyan Sun ${ }^{3}$ \\ Lin Nong (D) \\ Mangju Wang' \\ Xinan Cen' \\ 'Department of Hematology, Peking \\ University First Hospital, Beijing, People's \\ Republic of China; ${ }^{2}$ Xiamen \\ Cardiovascular Hospital, Xiamen \\ University, Xiamen, People's Republic of \\ China; ${ }^{3}$ Department of Respiration, \\ Peking University First Hospital, Beijing, \\ People's Republic of China; ${ }^{4}$ Department \\ of Pathology, Peking University First \\ Hospital, Beijing, People's Republic of \\ China
}

*These authors contributed equally to this work

Correspondence: Mangju Wang; Xinan

Cen

Department of Hematology, Peking

University First Hospital, No. 8 Xi Shi Ku

Street, Xi Cheng District, Beijing, I00034,

People's Republic of China

Tel +86-I0-83575746

Email wang_m_j@163.com;

cenxn@bjmu.edu.cn
Background: Myelomatous pleural effusion (MPE), as a presentation of extramedullary infiltration of multiple myeloma (MM), is rare and currently associated with poor outcomes without effective therapy. The potential value of cytokine detection in pleural effusion to MPE has not been reported to date.

Case Presentation: We herein report a case of refractory and relapsed multiple myeloma that developed bilateral MPE due to disease progression caused by intolerance to various chemotherapy regimens. Cytomorphology and flow cytometry were adopted for diagnosis confirmation. Chemotherapy containing immunomodulators combined with thoracic catheterization drainage was applied to the patient, showing a certain therapeutic effect. During the course of disease, the change of cytokine profile in pleural effusion was monitored by cytometric bead array (CBA) technology, revealing that cytokines related to tumor load such as interleukin 6 (IL-6) and interleukin 10 (IL-10) in pleural effusion decreased with the improvement of disease, while other cytokines such as interleukin 2 (IL-2), interleukin 4 (IL4), interleukin 17A (IL-17A), tumor necrosis factor $\alpha$ (TNF- $\alpha$ ), interferon $\gamma$ (IFN- $\gamma$ ), granzyme A, granzyme B, perforin and granulysin increased with the improvement of disease.

Conclusion: There is a prospect that cytokine level in pleural effusion may indicate treatment response of MPE, and in light of this case, immunomodulators may be utilized in treating patients suffering MPE. Due to limitations of our single case, we urge more groups to evaluate the potential role of cytokine profile in MPE.

Keywords: myelomatous pleural effusions, cytokine, IFN- $\gamma$, IL-2, immunomodulator

\section{Background}

$\mathrm{MM}$ is characterized by abnormal proliferation of monoclonal plasma cells secreting large amounts of monoclonal immunoglobulin or light chains inside bone marrow and finally leads to anemia, hypercalcemia, bone destruction, kidney injury, etc. Incidence of pleural effusion in MM patients is approximately $6 \%,{ }^{1}$ and the possible mechanisms include hypoalbuminemia, congestive heart failure caused by amyloidosis cardiomyopathy, renal dysfunction, pleural invasion, etc. ${ }^{2}$ Among them, we name the pleural effusion caused by pleural invasion as MPE, which is a type of extramedullary invasion with poor prognosis and treatment response. ${ }^{1}$ Other than tuberculous pleural effusion, a high level of adenosine deaminase (ADA) was also reported in one-third of MPE, which indicates MPE patients' varied immunocompetence based on ADA's important role in lymphatic system differentiation and maturation. ${ }^{3}$ In some cases, reports of chemotherapy combined with intrathoracic injection of IL-2 or interferon $\alpha$ (IFN- $\alpha$ ) attained a proven curative effect, achieving a remission duration of up to 30 months. ${ }^{4,5}$ All these 
phenomena imply that the immune system plays an important role in the development of MPE. We applied CBA technology (LEGENDplex ${ }^{\mathrm{TM}}$ Human CD8/NK Panel) to monitor cytokine profile in pleural effusion to explore the important role of cytokines during the disease course of an MPE patient.

The LEGENDplex ${ }^{\mathrm{TM}}$ Human CD8/NK Panel (Biolegend, cat.740267) is a multiplex bead-based assay, using fluorescence-encoded beads suitable for use on various flow cytometers. This panel allows simultaneous quantification of 13 human proteins with high detection sensitivities and broad dynamic ranges, including IL-2, IL4, IL-10, IL-6, IL-17A, TNF- $\alpha$, soluble Fas (sFas), soluble Fas Ligand (sFasL), IFN- $\gamma$, granzyme A, granzyme B, perforin and granulysin.

\section{Case Presentation}

The patient was a 70-year-old female who visited Beijing Hospital in 2014 for weakness, amaurosis and profuse sweating. The hemoglobin concentration was $63 \mathrm{~g} / \mathrm{L}$ $(6.3 \mathrm{~g} / \mathrm{dL})$. The white blood cell count was $6.49 \times 10^{9} / \mathrm{L}$. The platelet count was $100 \times 10^{9} / \mathrm{L}$. The laboratory chemistry profile showed total protein $110 \mathrm{~g} / \mathrm{L}$, albumin $36 \mathrm{~g} / \mathrm{L}$, calcium $2.32 \mathrm{mmol} / \mathrm{L}$, creatinine $74 \mathrm{umol} / \mathrm{L}$. The immunoglobulin profile showed IgA $80 \mathrm{~g} / \mathrm{L}, \operatorname{IgG} 4.15 \mathrm{~g} / \mathrm{L}, \operatorname{IgM}$ $0.04 \mathrm{~g} / \mathrm{L}$. The serum and urine immunofixation electrophoresis demonstrated IgA-Kappa (IgA- $\kappa)$ monoclonal gammopathy and the $\beta 2$-microglobulin was $8.89 \mathrm{mg} / \mathrm{L}$. Bone marrow aspiration showed monotypic plasma cells accounted for $60 \%$. The final diagnosis was IgA- $\kappa$ type multiple myeloma, International staging system (ISS) III and Durie-Salmon stage (D-S) IIIB. She had no significant medical history other than a myomectomy. She consecutively received six cycles of chemotherapy with different combinations of drugs, containing bortezomib, thalidomide, lenalidomide, ixazomib, cyclophosphamide, and dexamethasone. But this patient still suffered repeated disease progress due to drug intolerance. In November 2019, she was diagnosed with relapsed and refractory MM ( $\mathrm{r} / \mathrm{rMM})$. Daratumumab was then initiated. After a transient improvement, the disease progressed again in May 2020, impelling bortezomib to be used again. In June 2020, the patient developed bilateral pleural effusion when the frequency of daratumumab was reduced (Figure 1A, June 8, 2020, chest Computerized Tomography (CT)). Diagnostic thoracentesis showed bloody effusion with features suggestive of an exudate (specific gravity 1.030, nucleated cells count
$11,530 / \mathrm{mm}^{3}$, mononuclear cell $88 \%$, total protein $36.7 \mathrm{~g} /$ $\mathrm{L}$, albumin $23.5 \mathrm{~g} / \mathrm{L}$, lactate dehydrogenase (LDH) $463 \mathrm{U} /$ L, ADA 40.1 U/L). Microbiological examination including fungus and mycobacterium tuberculosis did not reveal any pathogens, and the next-generation sequencing (NGS) technology disclosed no pathogen either. Cytological analysis and flow cytometry analysis revealed a large number of abnormal plasma cells. On June 9, 2020, we detected the cytokine profile in peripheral blood and right-sided pleural effusion using CBA technology and the results are listed in Table 1. Subsequently, consecutive thoracic drainage was performed in combination with Daratumumab, liposomal Adriamycin, Thalidomide and Dexamethasone (DATD). The right-sided pleural effusion then decreased after 1 week. On June 15, 2020, we detected the cytokine profile in right-sided pleural effusion second time and the results are listed in Table 1. Due to the DATD intolerance, the chemotherapy regimen was changed to pomalidomide and dexamethasone. The disease remained stable. In July 2020, patient ceased taking pomalidomide due to myelosuppression. As a result, the left-sided pleural effusion began to increase slowly (Figure 1B: July 15, 2020, chest CT). The patient developed dyspnea in August 2020 with chest CT (Figure 1C, August 17, 2020) signifying the obvious increase of leftsided pleural effusion. Again, a microbiological examination of left-sided effusion found no pathogens. Cytological analysis and flow cytometry analysis revealed a large number of abnormal plasma cells. On August 17, 2020, the cytokine profile in left-sided pleural effusion was detected by CBA technology, and the results are listed in Table 1. The chemotherapy of liposomal Adriamycin plus dexamethasone was chosen together with respiratory support, left-sided thoracic catheterization drainage and antibiotics therapy. However, after a temporary improvement, the patient's disease continuously progressed. Chest CT was examined in September (Figure 1D, September 3, 2020), disclosing ground-glass opacity and consolidation in the right lung, diffuse ground-glass opacity in the left lung, the progression of lesions in upper lobe and decrease of bilateral pleural effusion. The patient died of exacerbation of pulmonary infection and respiratory failure on September 8, 2020.

\section{Discussion}

When MPE occurs as the initial symptom of MM, it is easy to be misdiagnosed as tuberculous (TB) pleural effusion because both are exudative and around one-third of 


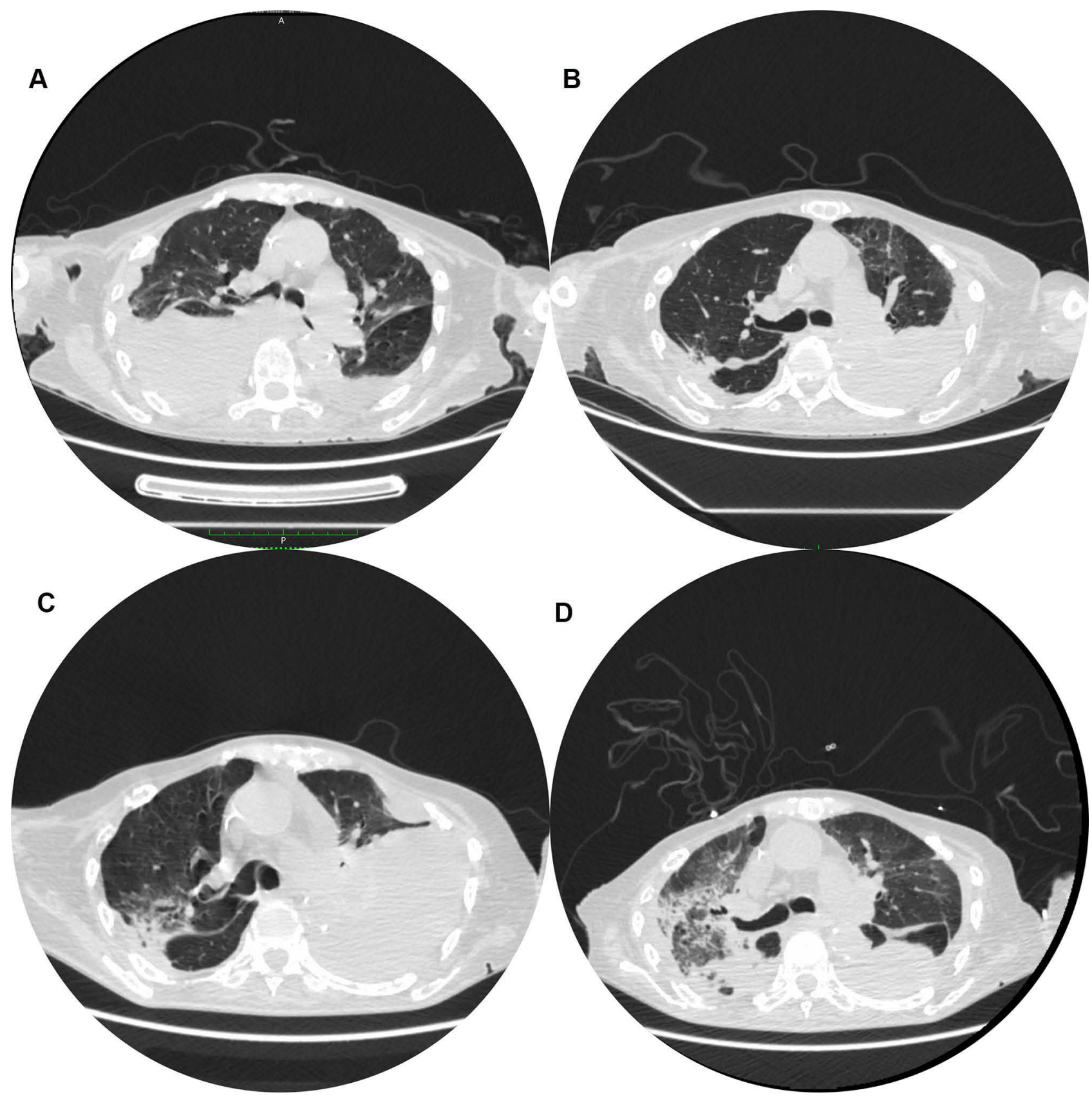

Figure I Changes in chest CT findings during the course of the disease. (A) Taken on 8 June 2020, Initial stage: Chest CT showed bilateral pleural effusion as well as left interlobar effusion and no manifestation of acute inflammation and space-occupying lesions. (B) Taken on I5 July 2020, Remission stage: slowly increasing tendency of the left pleural effusion and no increasing in right pleural effusion. (C) Taken on 17 August 2020, Relapse stage: obvious increase of left pleural effusion and decrease of right pleural effusion. (D) Taken on 3 September 2020, Terminal stage: Ground-glass opacity and consolidation in the right lung, diffuse ground-glass opacity in the left lung, the progression of lesions in upper lobe and decrease of bilateral pleural effusion.

MPE also have a higher ADA level. ${ }^{1}$ Furthermore, a certain proportion of myeloma plasma cells look like lymphocytes due to their intrinsic cytomorphological heterogeneity. The sensitivity of purified protein derivative (PPD) test, T-spot TB, mycobacterium tuberculosis culture, polymerase chain reaction (PCR) and NGS technology to make the diagnosis of tuberculosis pleural effusion is comparatively low. ${ }^{6}$ The invasive nature imposes restriction on the application of pleural biopsy, although its sensitivity has been proven. The ADA is not suitable as a differentiating criterion due to its increased level in MPE although it has both high sensitivity and specificity. ${ }^{6}$ It has been reported that IFN- $\gamma$ is significantly higher in TB pleural effusion than in malignant patients. $^{7}$ The 


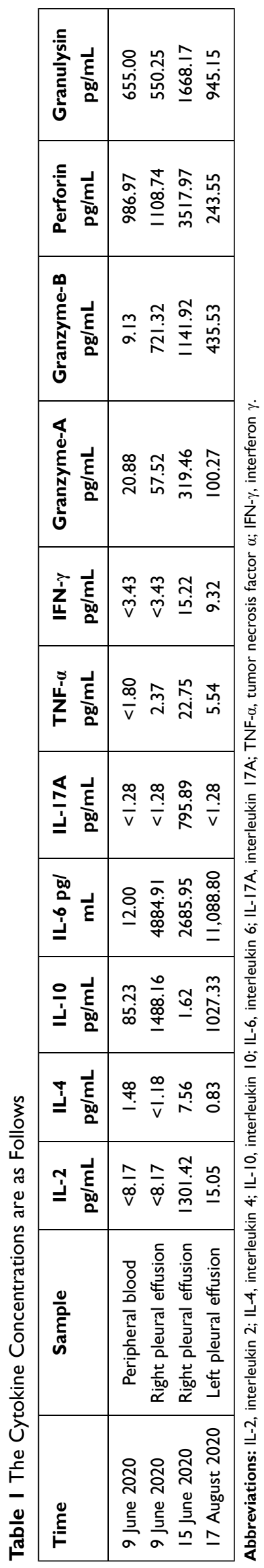

sensitivity and specificity of IFN- $\gamma$ over $140 \mathrm{pg} / \mathrm{mL}$ in differentiating the two kinds of pleural effusion are reported to be as high as $89-99 \%$ and $92-98 \%$, respectively. ${ }^{6}$ As a distinctive cytokine of Th1 cells, IFN$\gamma$ in peripheral blood and pleural effusion is significantly elevated by activated macrophages after the infection. Nevertheless, this patient was immunosuppressed; thus, her IFN- $\gamma$ level either in peripheral blood or pleural effusion was below the measurable range. There may be a great difference of IFN- $\gamma$ level between MPE and tuberculous pleural effusion, which drew a large prospect for IFN- $\gamma$ to be used in identification. Various cytokines interact with each other as a network, so their ratio may better define the pleural effusion's nature than the absolute concentration. The ratio of IL-4/IL-5, IL-4/TNF- $\alpha$, IL-2/TNF$\alpha$, and IL-10/TNF- $\alpha$ is lower and IFN- $\gamma / \mathrm{IL}-2, \mathrm{IL}-4, \mathrm{IL}-5$, IL-10, TNF- $\alpha$ in TB pleural effusion is higher than non-TB pleural effusion. ${ }^{7}$ However, whether the ratio of these cytokines can be used as a differentiating index needs larger sample size to verify further. The next question is whether cytokines can be used to distinguish MPE from other malignant pleural effusion and miscellaneous pleural effusion. Different cytokine-pairs with significant correlation were observed in different kinds of pleural effusion. ${ }^{7}$ The correlation between IFN- $\gamma$ and IL- 2 or IL-4 is unique in malignant pleural effusion, and correlation between TNF- $\alpha$ and IL-10 or IL-4 only exists in miscellaneous ones. ${ }^{7}$ Similarly, in our patient we revealed that the concentration of IL-2, IL-4, IL-17A, TNF- $\alpha$, IFN- $\gamma$, granzyme $\mathrm{A}$, granzyme $\mathrm{B}$, perforin and granulysin elevated simultaneously when MPE went into remission, which indicates a positive correlation among these cytokines. In contrast, IL-6 and IL-10 decreased at the same time. In MPE, there may be a specific negative correlation between the increased cytokines and decreased ones. However, whether this negative correlation can distinguish MPE from other types of pleural effusion requires further study. Nevertheless, cytokine profile in pleural effusion may provide potential biomarkers when it is difficult for routine methods to make the distinguishment.

MPE is usually an advanced manifestation of MM and its median survival time is only $2.8-4.0$ months even under active treatment. ${ }^{1}$ The lack of Th1 cytokines such as IL-2 as a sign of impaired or depressed fetal/neonatal immune response has been demonstrated to be significantly related to the prevalence of adverse outcomes in fetal pleural effusion. ${ }^{8}$ At present, multi-drug chemotherapy combined with repeated thoracentesis drainage or 
pleurodesis is strongly recommended. ${ }^{1}$ The right-sided pleural effusion of this patient was significantly reduced after the application of DATD regimen combined with thoracentesis drainage. Moreover, no progress occurred during maintenance treatment of pomalidomide. We revealed concentration of cytokines in pleural effusion is closely related to disease deterioration and improvement. The changes in these cytokines may represent the strength of the immune status in the chest cavity. The detection of cytokines in pleural effusion may become a barometer to predict the variation tendency of pleural effusion.

IL-2 is an important immunoregulatory cytokine in various diseases. ${ }^{9}$ A previous study reported that chemotherapy combined with IL-2 immunotherapy showed good curative effect in two cases of MPE with overall survival of up to 30 months. ${ }^{4}$ The lack of IL-2 was observed in fetal pleural effusion because of fetuses' impaired or depressed immune system. ${ }^{8}$ Our patient showed a higher IL-2 concentration in remission of pleural effusion and a lower IL-2 level in relapse, which indicated the potential value of IL-2 in MPE control. Due to the fact that IL-2 down-regulates the expression level of programmed cell death protein 1 (PD-1) and promotes the secretion of both granzyme B and IFN - $\gamma$, it can enhance tumor killing ability. ${ }^{9}$ IL-2 reverses $\mathrm{T}$ cell exhaustion based on these biological activities, which is probably related to its positive effect in treatment. In our case, both granzyme B and IFN- $\gamma$ in pleural effusion increased in pace with IL-2's increase, which fully verified this mechanism. However, more evidence is required to determine whether IL-2 can treat MPE. Additionally, which medication drives the cytokine variation in pleural effusion? Immunomodulators are suspected to be the answer to a large extent. Immunomodulators, including thalidomide, lenalidomide and pomalidomide, can induce $\mathrm{T}$ lymphocytes to secrete IL-2 through cereblon (CRBN) signaling pathway, and further inhibit the proliferation of myeloma cells, ${ }^{10}$ which is capable to state the reason why IL-2 returned to its initial level and why the pleural effusion significantly progressed after discontinuation of immunomodulators. Therefore, immunomodulators may inhibit the formation of pleural effusion through regulating immune environment inside thoracic cavity. As we all know, MPE is a special subtype of extramedullary disease (EMD). The effectiveness of different kinds of immunomodulators in EMD has been discussed in the published literature. No treatment response was observed in MM patients with extramedullary disease when taking thalidomide. ${ }^{11,12}$ Although it is considered that lenalidomide is a more potentially effective immunomodulator than thalidomide, there were few published data on the effectiveness of lenalidomide. ${ }^{13,14}$ A retrospective study demonstrated the efficacy and tolerability of pomalidomide/cyclophosphamide/dexamethasone combination in a group of patients with EMD. ${ }^{15}$ Our case showed the patient's disease remained stable during the use of pomalidomide. After stopping pomalidomide, her pleural effusion progressed again. It seemed immunomodulators may play a certain role in the control of MPE. Therefore, we suggest that immunomodulators may be utilized in treating patients suffering MPE.

\section{Conclusion}

To date, this is the first report describing the potentially important role of cytokines in myeloma pleural effusion. Our case indicates that a possible value of IFN- $\gamma$ to be applied in differentiating tuberculous pleural effusion from MPE. In addition, the concentration of various cytokines in pleural effusion may be used as a potential barometer to forecast the outcome of MPE. Finally, our work may provide evidence to include immunotherapy in the treatment of MPE. We are collecting more patients with MPE to perform cytokine measurements. We anticipate more valuable results and findings in the future. We also urge more groups to further evaluate the potential role of cytokine profile in MPE.

\section{Data Sharing Statement}

All data generated or analysed during this study are included in this published article.

\section{Ethics Approval and Consent to Participate}

Institutional approval was not required to publish the case details.

\section{Consent for Publication}

This patient's relatives provided written informed consent for the publication of the case details and images.

\section{Acknowledgments}

We thank her close relatives for allowing us to publish his case. 


\section{Author Contributions}

All authors made a significant contribution to the work reported, whether that is in the conception, study design, execution, acquisition of data, analysis and interpretation, or in all these areas. All authors took part in drafting, revising or critically reviewing the article; gave final approval for the version to be published; have agreed on the journal to which the article has been submitted; and have agreed to be accountable for all aspects of the work.

\section{Funding}

This study was funded by the Beijing Municipal Science Technology Commission (No. Z191100006619026). The funder had no role in the study except for financial support.

\section{Disclosure}

The authors declare that they have no conflicts of interest.

\section{References}

1. Yanamandra U, Deo P, Sahu KK, et al. Clinicopathological profile of myelomatous pleural effusion: single-center real-world experience and review of literature. Clin Lymphoma Myeloma Leuk. 2019;19:183189.e1. doi:10.1016/j.clml.2018.12.003

2. Kintzer JS, Rosenow EC, Kyle RA. Thoracic and pulmonary abnormalities in multiple myeloma. A review of 958 cases. Arch Intern Med. 1978;138:727-730. doi:10.1001/archinte.1978.03630290039015

3. Cho YU, Chi HS, Park CJ, Jang S, Seo EJ, Suh C. Myelomatous pleural effusion: a case series in a single institution and literature review. Korean J Lab Med. 2011;31:225-230.

4. Zhong Y, Zhang J, Wang H. Myelomatous pleural effusion involvement in 23 patients with multiple myeloma: a single-center clinical analysis. Thorac Cancer. 2015;6:359-362. doi:10.1111/1759-7714.12220

5. Makino S, Yamahara S, Nagake Y, Kamura J. Bence-Jones myeloma with pleural effusion: response to alpha-interferon and combined chemotherapy. Int Med. 1992;31:617-621. doi:10.2169/internalmedicine.31.617
6. Villena Garrido V, Cases Viedma E, Fernández Villar A, et al. Recommendations of diagnosis and treatment of pleural effusion. Update. Arch Bronconeumol. 2014;50:235-249. doi:10.1016/j.arbr. 2014.04.007

7. Aoe K, Hiraki A, Murakami T, et al. Relative abundance and patterns of correlation among six cytokines in pleural fluid measured by cytometric bead array, Int. J Mol Med. 2003;12 (2):193-198.

8. Imai K, Kotani T, Tsuda H, Kobayashi T, Ushida T, Moriyama Y. Determination of the cytokine levels in fetal pleural effusion and their association with fetal/neonatal findings. Cytokine. 2020;127:154945. doi:10.1016/j.cyto.2019.154945

9. Han L, Jiang Q, Yao W, Fu T, Zeng Q. Thoracic injection of low-dose interleukin-2 as an adjuvant therapy improves the control of the malignant pleural effusions: a systematic review and meta-analysis base on Chinese patients. BMC Cancer. 2018;18:725. doi:10.1186/ s12885-018-4581-5

10. Lindner S, Krönke J. The molecular mechanism of thalidomide analogs in hematologic malignancies. J Mol Med. 2016;94:13 27-1334. doi:10.1007/s00109-016-1450-z

11. Rosiñol L, Cibeira MT, Bladé J, et al. Extramedullary multiple myeloma escapes the effect of thalidomide. Haematologica. 2004;89:832-836.

12. Wu P, Davies F, Boyd K, et al. The impact of extramedullary disease at presentation in the outcome of myeloma. Leuk Lymphoma. 2009;50:230-235. doi:10.1080/10428190802657751

13. Richardson PG, Blood E, Mitsiades CS, et al. A randomized Phase 2 study of lenalidomide therapy for patients with relapsed or relapsed and refractory multiple myeloma. Blood. 2006;108:3458-3464. doi:10.1182/blood-2006-04-015909

14. Kumar S, Flinn IW, Richardson PG, et al. Novel three- and four-drug combination regimens of bortezomib, dexamethasone, cyclophosphamide, and lenalidomide, for previously untreated multiple myeloma: results from the multicenter, randomized, phase 2 EVOLUTION study. Blood. 2006;116:621(abstr 621).

15. Hagihara M, Ide S, Ohara S, Uchida T, Inoue M, Hua J. omalidomide/cyclophosphamide/dexamethasone combination therapy for relapsed/refractory multiple myeloma accompanied by extramedullary. lesions Rinsho Ketsueki. 2020;61(1):20-26.
OncoTargets and Therapy

\section{Publish your work in this journal}

OncoTargets and Therapy is an international, peer-reviewed, open access journal focusing on the pathological basis of all cancers, potential targets for therapy and treatment protocols employed to improve the management of cancer patients. The journal also focuses on the impact of management programs and new therapeutic agents and protocols on patient perspectives such as quality of life, adherence and satisfaction. The manuscript management system is completely online and includes a very quick and fair peer-review system, which is all easy to use. Visit http://www.dovepress.com/ testimonials.php to read real quotes from published authors. 